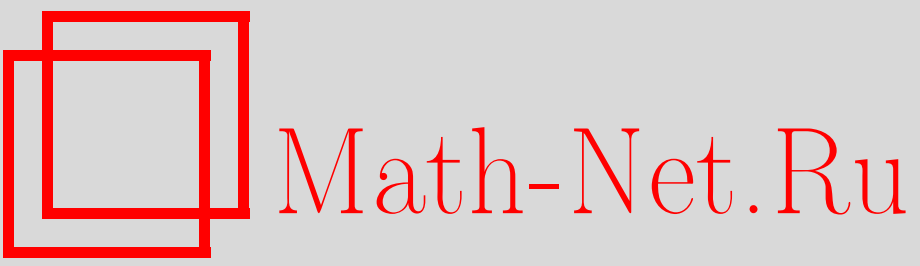

Т. М. Суидан, Одномерный гравитационно взаимодействующий газ и выпуклая миноранта броуновского движения, УМН, 2001, том 56, выпуск 4, 73-96

DOI: https://doi.org/10.4213/rm416

Использование Общероссийского математического портала Math-Net.Ru подразумевает, что вы прочитали и согласны с пользовательским соглашением

http://www.mathnet.ru/rus/agreement

Параметры загрузки:

IP : 54.198 .64 .247

26 апреля 2023 г., 17:54:40 


\title{
ОДНОМЕРНЫЙ ГРАВИТАЦИОННО ВЗАИМОДЕЙСТВУЮШИЙ ГАЗ И ВЫПУКЛАЯ МИНОРАНТА БРОУНОВСКОГО ДВИЖЕНИЯ
}

\author{
Т. М. СУИДАН
}

Эта статья посвящена удивительной связи между динамикой одномерного гравитационно взаимодействующего газа частиц с прилипанием и выпуклой минорантой броуновского движения на $[0,1]$. Мы изучаем эту динамику, устанавливая три различных кластеризующих режима и временнь́е масштабы, на которых они происходят. Распределение масс газа в критический момент времени может быть вычислено в терминах функционалов от процесса выпуклой миноранты.

Библиография: 16 названий.

\section{СОДЕРЖАНИЕ}

1. Введение

2. Выпуклые миноранты случайных блужданий и броуновского движе-

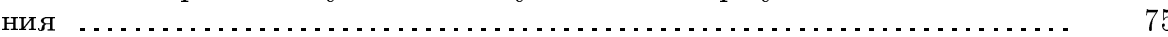

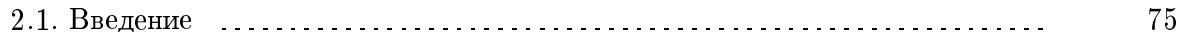

2.2. Самый длинный отрезок выпуклой миноранты случайного блуждания

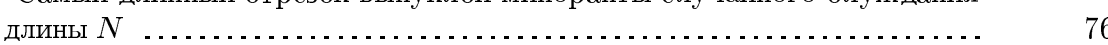

2.3. Выпуклая миноранта броуновского движения ................... 77

2.4. Число отрезков, составляющих вьпуклую миноранту случайного блуждания длины $N$

3. Динамика с прилипанием и случайные блуждания …................ 82

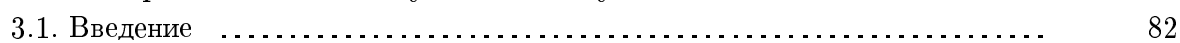

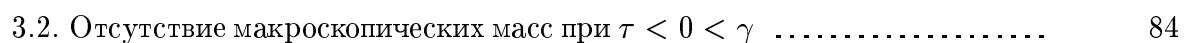

3.3. Агрегация масс в критический момент времени $\tau=0 \quad \ldots \ldots \ldots \ldots \ldots \ldots . \ldots . \ldots$

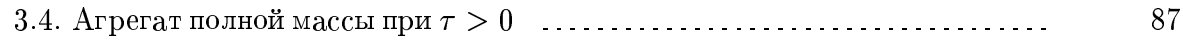

3.5. Равномерный контроль предельного процесса: последовательность моментов времени ....................................... 88

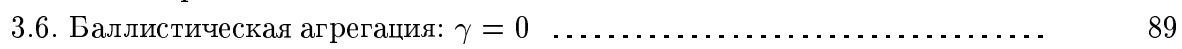

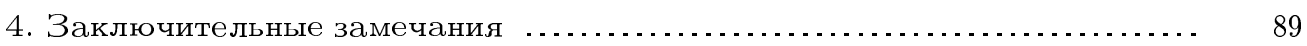

5. Приложение ....................................................... 90

5.1. Некоторые теоремы о конечных последовательностях вещественных чи-

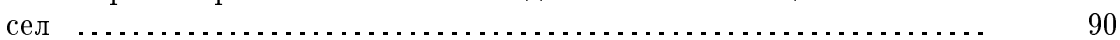

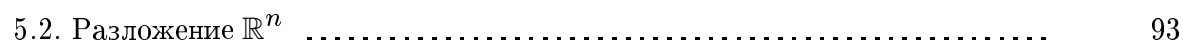

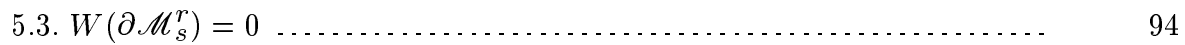

5.4. Неравенство Марцинкевича-Зигмунда и болшшие отклонения . . . . . . . . 94

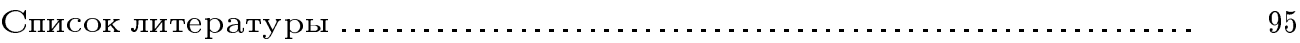




\section{1. Введение}

Статья состоит из двух частей. Первая часть (раздел 2) содержит анализ вьпуклой миноранты броуновского движения на конечном временно́м интервале. В частности, мы изучаем совместные распределения длин $r$ самых длинных сегментов вьпуклой миноранты. Во второй части (раздел 3 ) мы используем эти распределения для анализа упрошенной астрофизической модели, предложенной Зельдовичем [16] для изучения неравномерного распределения масс во вселенной. Конкретная формулировка задачи и изучаемая здесь модель принадлежат Филипп Мартену и Ярославу Пясецки [10]. Они поставили и затем изучили следуюший вопрос: пусть дано $N$ равномерно распределенных одинаковых частиц на единичном отрезке $[0,1] \subset \mathbb{R}$ с независимыми одинаково распределенньми гауссовыми начальньми скоростями и с вполне неупругим законом столкновения и одномерной гравитацией; что можно сказать об их динамике? В точной постановке, гамильтониан, описьваюший динамику между столкновениями, имеет вид

$$
H_{N}=\sum_{i=1}^{N} \frac{p_{i}^{2}}{2 m_{i}}+\gamma \sum_{i<j} m_{i} m_{j}\left|x_{i}-x_{j}\right|
$$

где $\gamma$ - гравитационная постоянная, $m_{i}, x_{i}$ и $p_{i}$ - масса, положение и импульс частицы $i$, а потенциалом является стандартный одномерный гравитационный потенциал. Столкновения считаются вполне неупругими, при которых сохраняются импульс и масса, но не энергия.

У описанной задачи есть связь с трехмерной задачей Зельдовича: можно рассматривать частицы и процесс их агрегации как результат равномерного распределения масс, при котором образуются галактики во вселенной (кластеры масс, разделенные бескрайними просторами). С другой стороны, одномерная гравитация отличается от трехмерной гравитации, да и одномерньй закон столкновений тоже.

Вот утверждение, доказанное Мартеном и Пясецки [10]. Существует неслучайное время, $t^{*}$, не зависящее от второго момента гауссова распределения скоростей, такое, что в непрерьвном пределе (при $N \rightarrow \infty$ и $m_{i} \rightarrow 0$ так, что $m_{i} N=M=$ const) вьполняется следующее утверждение: с вероятностью 1 для любого фиксированного момента времени $t<t^{*}$ макроскопические массы не образуются; с вероятностью 1 для любого фиксированного момента времени $t>t^{*}$ существует одна макроскопическая масса $M$. Грубо говоря, макроскопическая масса - это агрегат, содержаший конечную долю полной массы системы. Иначе говоря, этот критический неслучайный момент времени разделяет два различных режима агрегации масс. В разделе 3 эта задача изучается в случае, когда начальные распределения скоростей не обязательно гауссовы; мы показываем, что упомянутое вьше поведение универсально в том смысле, что оно применимо к большому классу начальных распределений скоростей. В частности, единственное требуемое свойство состоит в том, чтобы начальное распределение скоростей имело моменты порядка $4+\delta$ для некоторого $\delta>0$. Мы воспроизводим результаты Мартена и Пясецки и приводим более сильные результаты о размере масс перед критическим моментом времени. Наши методы отличаются от использованных в [10] и учитьвают флуктуации случайных сумм. Наиболее интересна динамика в критический момент. Она подробно исследуется, для чего процесс агрегации реализуется в терминах случайных блужданий и связанных с ними выпуклых 
минорант. Этот подход позволяет высказьвать содержательные утверждения о критическом моменте времени в непрерьвном пределе, изучая вьпуклую миноранту броуновского движения. В этом анализе используются вычисления, включаюшие асимптотическое поведение функционалов от случайных перестановок.

Автор выражает признательность Я. Г. Синаю за помощь и поддержку. Автор также благодарит А. Бородина, Р. Кармона, В. И, Ч. Голди, Я. Пясецки, Дж. Питмана, М. Стили и М. Йора за полезные обсуждения.

\section{2. Выпуклые миноранты случайных блужданий и броуновского движения}

2.1. Введение. В этом разделе мы обсуждаем свойства вьпуклых минорант случайных блужданий, порожденных вещественными независимыми одинаково распределенньми случайными величинами, имеюшими плотности. Вьпуклая миноранта любой непрерьвной функции $f$ на отрезке $[0, t]$ определяется как наибольшая выпуклая функция $g$ на $[0, t]$, удовлетворяющая неравенству $g \leqslant f$. Перечислим основные результаты этого раздела.

(i) Для случайного блуждания длины $N$ вероятность того, что соответствующая вьпуклая миноранта содержит отрезок длины $n$, равна $\frac{1}{n}$ при $n>\frac{N}{2}$. Для любого $n \leqslant N$ математическое ожидание числа отрезков длины $n$ равно $\frac{1}{n}$.

(ii) При $s \in[0,1]$ и для любого натурального $r$ вероятность того, что длина $\mathscr{L}_{W}^{r}$ $r$-го по длине отрезка вьпуклой миноранты броуновского движения на $[0,1]$ меньше числа $s$, равна $F_{r}(s)$, где $F_{r}(s)$ задается явной формулой; совместная плотность $p_{r}\left(x_{1}, \ldots, x_{r}\right)$ для набора длин $r$ самых длинных отрезков может быть выражена явно через элементарные функции. Математическое ожидание числа отрезков длины $l \mathrm{c}$ $1 \geqslant t \geqslant l \geqslant s>0$ равно $\log \frac{t}{s}$.

(iii) Для случайного блуждания длины $N$ вероятность того, что отвечающая этому блужданию вьпуклая миноранта составлена из $m$ отрезков, равна

$$
\left.\frac{1}{m ! N !} \frac{d^{N}}{d z^{N}}(-\log (1-z))^{m}\right|_{z=0}
$$

Математическое ожидание числа этих отрезков равно $\sum_{i=1}^{N} \frac{1}{i}$. Дисперсия числа отрезков равна $\sum_{i=1}^{N}\left(\frac{1}{i}-\frac{1}{i^{2}}\right)$.

В каждом из этих утверждений под длиной отрезка понимается длина проекщии на $[0, N]$ или $[0,1]$ в зависимости от контекста. Очевидно, случайное блуждание может иметь не более одного отрезка длины $n>\frac{N}{2}$. Поэтому формула в (i) для вероятности может быть применена и к длине самого длинного отрезка выпуклой миноранты. Чарльз Голди указал нам, что утверждение (iii) было доказано другими методами Спарре Андерсеном [13]. 
Раздел организован следуюшим образом. В п. 2.2 мы доказьваем утверждение (i). В п. 2.3 мы используем случайные перестановки и замечательные асимптотические результаты Шеппа-Ллойда [12] и Вершика-Шмидта [15] для доказательства утверждения (ii). В п. 2.4 мы доказываем (iii) и обнаруживаем связь с числами Стирлинга первого рода. Важно отметить, что от случайных величин порождаюших рассматриваемые нами случайные блуждания мы не требуем существования первых моментов, за исключением п. 2.3, где мы требуем существования вторых моментов. Кроме того, результаты этого раздела справедливы и в случае, когда случайные величины, порождаюшие случайные блуждания, имеют непрерывные распределения, не обязательно допускаюшие плотности; однако для простоты изложения мы формулируем результаты для случайных величин в предположении, что плотности у них есть. В этом анализе мы используем некоторые теоремы, доказанные в приложении. Эти теоремы касаются свойств последовательностей вешественных чисел и их вероятностных следствий, а также полезного разложения пространства $\mathbb{R}^{n}$. В частности, мы используем следуюшее утверждение: вероятность того, что случайное блуждание длины $N$ остается над прямой, соединяющей начало координат с концевой точкой, равно $\frac{1}{N}$; приведен также условньй вариант этого утверждения. М. Стили указал нам, что Бакстер [3] и Спитцер [14] уже знали это утверждение.

2.2. Самый длинный отрезок выпуклой миноранты случайного блуждания длины $N$. Пусть $X_{1}, \ldots, X_{N}$ - вещественные независимые одинаково распределенные случайные величины с плотностями. Для $n>\frac{N}{2}$ мы вычислим сейчас вероятность $L_{n}$ того, что длина самого длинного отрезка вьпуклой миноранты, связанной со случайным блужданием, порожденным $X_{1}, \ldots, X_{N}$, равна $n$. Пусть $p_{n}(u)$ - плотность случайной величины $\frac{X_{1}+\cdots+X_{n}}{n}$.

TeOpema 2.2.1. $L_{n}=\frac{1}{n} n p u n>\frac{N}{2}$.

ДокАЗАТЕльство. Вероятность того, что отрезок длины $n$ начинается в точке с координатой $j \leqslant N-n$, равна

$$
\begin{aligned}
& \int_{\mathbb{R}} d u p_{n}(u)\{ \mathrm{P}\left(\sum_{l=1}^{i} X_{j+l}>u i, 1 \leqslant i<n \mid \frac{1}{n} \sum_{l=1}^{n} X_{j+l}=u\right) \\
& \times \mathrm{P}\left(\sum_{s=0}^{r} X_{j-s}<u(r+1), 0 \leqslant r<j\right) \\
&\left.\times \mathrm{P}\left(\sum_{t=1}^{m} X_{j+n+t}>u m, 1 \leqslant m \leqslant N-(j+n)\right)\right\} \\
&=\int_{\mathbb{R}} d u p_{n}(u) \frac{1}{n} K_{j}(u) G_{N-(n+j)}(u)
\end{aligned}
$$

где $K_{j}(u)$ и $G_{N-(n+j)}(u)$ - второй и третий сомножители в нашем произведении вероятностей. Первьй сомножитель равен $\frac{1}{n}$ в силу замечания в конще п. 2.1 ; доказательство этого факта приведено в приложении 5.1. Так как событие, состоящее в том, что 
отрезок вьпуклой миноранты, размер которого в точности равен $n>\frac{N}{2}$, начинается в точке с координатой $j$, и событие, состояшее в том, что такой отрезок начинается в точке с другой координатой $l$, несовместны, то вероятность того, что отрезок размера $n>\frac{N}{2}$ есть, равна

$$
\begin{aligned}
& \sum_{j=0}^{N-n} \int_{\mathbb{R}} d u p_{n}(u) \frac{1}{n} K_{j}(u) G_{N-(n+j)}(u) \\
& \quad=\frac{1}{n} \int_{\mathbb{R}} d u p_{n}(u) \sum_{j=0}^{N-n} K_{j}(u) G_{N-(n+j)}(u) \\
& \quad=\frac{1}{n} \int_{\mathbb{R}} d u p_{n}(u)=\frac{1}{n} .
\end{aligned}
$$

Здесь мы использовали соотношение $\sum_{j=0}^{N-n} K_{j}(u) G_{N-(n+j)}(u)=1$, доказанное в приложении 5.2 .

Заметим, что при $1 / 2<s<t \leqslant 1$ вероятность того, что имеется отрезок выпуклой миноранты длины $n$, где $s N \leqslant n \leqslant t N$, равна $\sum_{s N \leqslant n \leqslant t N} 1 / n$, а эта сумма стремится к $\log (t / s)$ при $N \rightarrow \infty$. Из доказательства теоремы 2.2.1 вытекает также следуюший факт.

СлЕДСТВИЕ 2.2.1. Для любого $n \in\{1, \ldots, N\}$ математическое оэсидание числа отрезков длины $n$ в выпуклой миноранте равно $1 / n$.

ДокАЗАТЕльство. Если не настаивать на несовместности событий на втором шаге доказательства теоремы 2.2.1, то наше вычисление дает выражение для математического ожидания числа отрезков длины $n$ выпуклой миноранты, связанной с рассматриваемьми случайными блужданиями.

2.3. Выпуклая миноранта броуновского движения. Начнем с напоминания замечательных результатов Шеппа-Ллойда [12] и Вершика-Шмидта [15] об асимптотическом распределении длин циклов в случайных перестановках. Обозначим через $\mathscr{S}_{n}$ симметрическую групу $n$ символов и введем на ней равномерное вероятностное распределение $\mathrm{P}$. Для $\pi \in \mathscr{S}_{n}$ обозначим через $\alpha_{i}(\pi), i=1,2, \ldots, n$, число циклов длины $i$ в $\pi$; набор $\left(\alpha_{1}(\pi), \ldots, \alpha_{n}(\pi)\right)$ называется цикловым типом $\pi$. Пусть $L_{r}^{n}(\pi)$ - длина $r$-го по длине цикла в $\pi \in \mathscr{S}_{n}$ (мы считаем, что $L_{r}^{n}(\pi)=0$, если число циклов в $\pi$ меньше $r$ ). Легко видеть, что

$$
\mathrm{P}\left(\pi \in \mathscr{S}_{n}: \alpha_{1}(\pi)=a_{1}, \ldots, \alpha_{n}(\pi)=a_{n}\right)=\prod_{j=1}^{n} \frac{(1 / j)^{a_{j}}}{a_{j} !} .
$$

Как мы увидим в следующем пункте, эта величина в точности равна вероятности того, что соответствующая вьпуклая миноранта случайного блуждания длины $n$ составлена из $a_{1}$ отрезков длины $1, a_{2}$ отрезков длины 2 , и т. д.; этот факт порождает естественную биекцию между цикловыми типами $\mathscr{S}_{n}$ и классами длин составляющих отрезков (без учета порядка) вьпуклой миноранты случайных блужданий длины $n$. 
Мы сейчас используем эту биекцию. На самом деле связь между этими двумя объектами имеет некоторую историю, отраженную в статье Голди [8]. В [12] доказано, что предельное распределение $L_{r}^{n} / n$ может быть описано явно с помошью следующей функции распределения $F_{r}(\xi)$ на $[0, \infty)$ :

$$
\begin{aligned}
& F_{r}(\xi)=1-\sum_{p=r}^{p<\xi^{-1}} \frac{(-1)^{p-r}}{(r-1) !(p-r) ! p} \int_{A_{p}(\xi)} \int \frac{d u_{1} \cdots d u_{p}}{u_{1} \cdots u_{p}} \text { при } 0<\xi<\frac{1}{r}, \\
& F_{r}(\xi)=1 \text { при } \frac{1}{r} \leqslant \xi<\infty,
\end{aligned}
$$

где $A_{p}(\xi)$ - область интегрирования, заданная неравенствами $u_{1}, \ldots, u_{p} \geqslant 1$ и $u_{1}+$ $\cdots+u_{p} \leqslant \xi^{-1}$. Мы можем сказать даже больше. В [15] доказано, что предельное распределение набора $\left(\frac{L_{1}^{n}}{n}, \ldots, \frac{L_{r}^{n}}{n}\right)$ сушествует и имеет плотность $p_{r}\left(x_{1}, \ldots, x_{r}\right)$ на множестве $\Lambda_{r}=\left\{x \in \mathbb{R}^{r}: x_{1} \geqslant \cdots \geqslant x_{r} \geqslant 0, \sum_{i=1}^{r} x_{i} \leqslant 1\right\}$, заданную формулой

$$
\frac{1}{x_{1} \cdots x_{r-1} \bar{x}_{r}} f\left(\frac{x_{r}}{\bar{x}_{r}}\right)
$$

где $f(\xi)=\frac{d F_{1}(\xi)}{d \xi}$ и $\bar{x}_{i}=1-x_{1}-\cdots-x_{i-1}$.

Напомним вкратце понятия броуновского движения, меры Винера и приближения Донскера. Пусть $C[0,1]$ - банахово пространство непрерьвных функций на $[0,1]$ со стандартной sup-нормой; обозначим через $\mathscr{C}$ борелевскую сигма-алгебру на $C[0,1]$ и через $W$ стандартную меру Винера на измеримом пространстве $(C[0,1], \mathscr{C})[4]$. Введем перенормированные процессы случайных блужданий $Y_{t}^{N}$ на $[0,1]$ формулой

$$
Y_{t}^{N}=\frac{S_{\lfloor N t\rfloor}+(N t-\lfloor N t\rfloor) X_{\lfloor N t\rfloor+1}}{\sqrt{N E X_{1}^{2}}},
$$

где $S_{i}=\sum_{j=1}^{i} X_{j}$, а $X_{1}, \ldots, X_{N}$ - такие же, как и вьше, и имеют вторые моменты. Теория слабой сходимости мер на метрических пространствах и приближение Донскера [4] упрощают вычисление некоторых интересных величин. Если $A \in \mathscr{C}$ и $W(\partial A)=0$, то $W_{N}(A) \rightarrow W(A)$ при $N \rightarrow \infty$; здесь $W_{N}$ обозначает меру на $(C[0,1], \mathscr{C})$ индуцированную перенормированными процессами случайных блужданий $Y_{t}^{N}$, а $\partial A$ обозначает границу $A$ в смысле топологии метрического пространства на $C[0,1]$.

Пусть $s \in[0,1]$. Обозначим через $\mathscr{M}_{r}^{s}$ множество таких функций $\omega \in C[0,1]$, что длина $r$-го по длине отрезка выпуклой миноранты $\omega$ принимает значение в $(0, s]$. Заметим, что $W\left(\partial \mathscr{M}_{r}^{s}\right)=0$; набросок доказательства этого факта приведен в приложении. Мы сейчас вычислим $W\left(\mathscr{M}_{r}^{s}\right)$, непосредственно вычисляя $\lim _{N \rightarrow \infty} W_{N}\left(\mathscr{M}_{r}^{s}\right)$. Из приведенного выше рассуждения о случайных перестановках и выпуклых минорантах и из простых масштабных соображений следует, что

$$
F_{r}(s)=\lim _{N \rightarrow \infty} \mathrm{P}\left(\frac{L_{r}^{N}}{N} \leqslant s\right)=\lim _{N \rightarrow \infty} W_{N}\left(\mathscr{M}_{r}^{s}\right)=W\left(\mathscr{M}_{r}^{s}\right),
$$

где $F_{r}$ определено вьше. Пусть $\mathscr{L}_{W}^{r}$ - длина $r$-го по длине отрезка выпуклой миноранты броуновского движения на $[0,1]$. Мы получаем требуемое утверждение: 
Tеорема 2.3.1. При $s \in[0,1]$ имеем $\mathrm{P}\left(\mathscr{L}_{W}^{r} \in[0, s]\right)=F_{r}(s)$, əде $F_{r}(\xi)=1$ nрu $\xi \geqslant 1 / r$ и $F_{r}(\xi)$ при $0<\xi<1 / r$ определяется формулой

$$
F_{r}(\xi)=1-\sum_{p=r}^{p<\xi^{-1}} \frac{(-1)^{p-r}}{(r-1) !(p-r) ! p} \int_{A_{p}(\xi)}^{\ldots} \int \frac{d u_{1} \cdots d u_{p}}{u_{1} \cdots u_{p}},
$$

а $A_{p}(\xi)$ определено выше.

Этот результат очевидным образом переносится на броуновское движение на любом конечном отрезке $[0, T], T<\infty$, и соответствуюшее распределение равно $F_{r}(\xi / T)$. Таким образом, мы полностью охарактеризовали вероятностное распределение длины $r$-го по длине отрезка вьпуклой миноранты броуновского движения на конечных временни́х отрезках. Это распределение принимает особенно красивую форму при $r=1$ и $s>1 / 2$, а именно, $F_{1}(s)=1+\log s$. Аналогично, можно использовать приближение Донскера и результаты [15] для доказательства следуюшей теоремы.

Теорема 2.3.2. Совместное распределение $\left(\mathscr{L}_{W}^{1}, \ldots, \mathscr{L}_{W}^{r}\right)$ имеет плотность $p_{r}\left(x_{1}, \ldots, x_{r}\right)$ на $\Lambda_{r}$, заданную формулой

$$
\frac{1}{x_{1} \cdots x_{r-1} \bar{x}_{r}} f\left(\frac{x_{r}}{\bar{x}_{r}}\right)
$$

əде $f(\xi)=\frac{d F_{1}(\xi)}{d \xi} u \bar{x}_{i}=1-x_{1}-\cdots-x_{i-1}$.

Этот результат полностью характеризует совместное распределение длин $r$ наиболее длинных отрезков вьпуклой миноранты на $[0,1]$. Как и вьше, этот результат допускает перенесение на броуновское движение на любом конечном отрезке $[0, T]$. Интересно отметить также, что, используя приближение Донскера и результаты п. 2.4, мы можем вычислить математическое ожидание числа отрезков вьпуклой миноранты броуновского движения, длины которых содержатся в $[s, t]$, где $0<s<t \leqslant 1$; это математическое ожидание равно $\log (t / s)$. Для доказательства мы должны только имитировать доказательство следствия 2.4.1.

Проблема выпуклой миноранты броуновского движения рассматривалась ранее (см., например, [2], [9], [11]). Настоящая работа посвящена исследованию других интересных особенностей этой задачи, в том числе - исследованию распределения последовательных наклонов и его связям с чисто точечными процессами.

2.4. Число отрезков, составляющих выпуклую миноранту случайного блуждания длины $N$. Пусть $X_{1}, \ldots, X_{N}$ - вещественные независимые одинаково распределенные случайные величины, имеющие плотности. Мы используем методы, развитые в предыдуших разделах и в приложении, чтобы доказать следуюший результат Спарре Андерсена [13].

Теорема 2.4.1. Вероятность того, что выпуклая миноранта, отвечающая случайному блужданию, порожденному $X_{1}, \ldots, X_{N}$, составлена в точности из $m$ отрезков, равна

$$
\frac{1}{m !} \sum_{\substack{n_{1}+\cdots+n_{m}=N \\ n_{1}, \ldots, n_{m}>0}} \frac{1}{n_{1} \cdots n_{m}}=\left.\frac{1}{m ! N !} \frac{d^{N}}{d z^{N}}\left\{\left(\log \frac{1}{1-z}\right)^{m}\right\}\right|_{z=0} .
$$


ДокАЗАтельство. Начнем с некоторых определений. Пусть $\mathbb{Z}_{m}^{N}=\left\{\left(b_{1}, \ldots, b_{m}\right)\right.$ $\left.\in \mathbb{Z}^{m}: b_{i}>0, b_{1}+\cdots+b_{m}=N\right\}$. Введем отношение эквивалентности $\delta$ на $\mathbb{Z}_{m}^{N}$ следующим образом: $\left(c_{1}, \ldots, c_{m}\right) \delta\left(b_{1}, \ldots, b_{m}\right)$ тогда и только тогда, когда $\left(c_{1}, \ldots, c_{m}\right)=$ $\tau\left(b_{1}, \ldots, b_{m}\right)$ для некоторого элемента $\tau$ симметрической группы порядка $m$. Обозначим через $\mathscr{H}$ факторпространство $\mathbb{Z}_{m}^{N} / \delta$. Определим функцию кратности $g$ на $\mathscr{H}$ следуюшим образом: если $b \in \mathscr{H}$, то $g(b)=\frac{1}{r_{1} ! r_{2} ! \cdots r_{j} !}$, где $r_{1}, \ldots, r_{j}-$ кратности координат набора $b$; например, если $N=20, m=7$ и один из представителей $b \in \mathscr{H}$ равен $(4,2,2,5,3,1,3)$, то $g(b)=\frac{1}{2 ! 2 !}=\frac{1}{4}$. Положим $P(b)=\frac{g(b)}{b_{1} \cdots b_{m}}$. Переписьвая левую часть формулы (2), мы получаем

$$
\begin{aligned}
\frac{1}{m !} \sum_{\substack{n_{1}+\cdots+n_{m}=N \\
n_{1}, \ldots, n_{m}>0}} \frac{1}{n_{1} \cdots n_{m}} & =\frac{1}{m !} \sum_{b \in \mathscr{H}} \frac{1}{b_{1} \cdots b_{m}}(m ! g(b)) \\
& =\sum_{b \in \mathscr{H}} \frac{1}{b_{1} \cdots b_{m}} g(b)=\sum_{b \in \mathscr{H}} P(b) .
\end{aligned}
$$

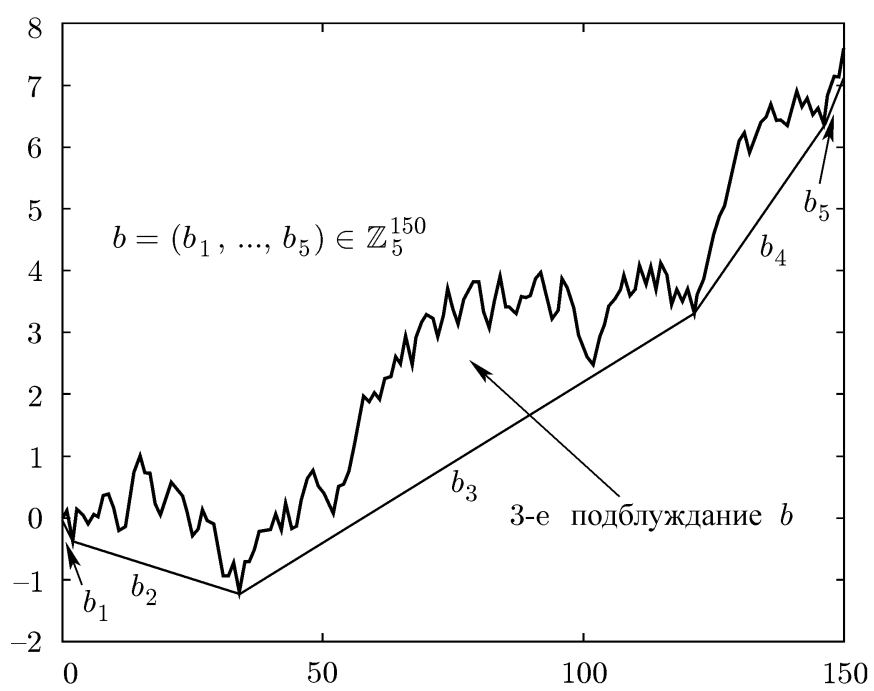

Рис. 1. Реализация случайного блуждания и ее выпуклая миноранта

Поясним роль функции $P(b)$ для $b \in \mathscr{H}$. Фиксируем $c \in \mathbb{Z}_{m}^{N}$ и определим $i$-е подблуждание $c$ как случайное блуждание длины $c_{i}$, начинающееся в точке с координатой $c_{1}+\cdots+c_{i-1}$ и заканчивающееся в точке с координатой $c_{1}+\cdots+c_{i}$; это - в точности часть случайного блуждания длины $N$, порожденная $X_{1+c_{1}+\cdots+c_{i-1}}, \ldots, X_{c_{1}+\cdots+c_{i}}$. Рис. 1 изображает одну из реализаций подобного случайного блуждания и его подблужданий. Пусть случайные величины $s_{1}^{c}, \ldots, s_{m}^{c}$ суть наклоны прямых, соединяющих концевые точки подблужданий точек $c$. Вероятность того, что каждое из $m$ подблужданий $c$ лежит вьше прямой, соединяюшей концевые точки этого 
подблуждания, и при этом $s_{1}^{c}<\cdots<s_{m}^{c}$, равна $\frac{1}{c_{1} \cdots c_{m}} \mathrm{P}\left(s_{1}^{c}<\cdots<s_{m}^{c}\right)$; мультипликативная структура связана с независимостью всех рассматриваемых событий; эта вероятность вычислена с учетом формул из приложения 5.1. Наши ограничения совпадают с условиями, что вьпуклая миноранта случайного блуждания длины $N$ составлена из $m$ отрезков с длинами $c_{1}, \ldots, c_{m}$; см. рис. 1 . Суммируя по различньм (с точки зрения порядка) перестановкам координат точки $c$, получаем

$$
\frac{g(c)}{c_{1} \cdots c_{m}}
$$

где на этот раз $c$ рассматривается как элемент $\mathscr{H}$, а не $\mathbb{Z}_{m}^{N}$. Полученное выражение для $P(c)$ равно вероятности того, что вьпуклая миноранта случайного блуждания длины $N$ содержит в точности $m$ отрезков с длинами $c_{1}, \ldots, c_{m}$, без учета порядка. Суммируя по всем $c \in \mathscr{H}$, мы получаем последнее выражение предыдущего вычисления и первое выражение в (1).

Это выражение можно непосредственно вычислить с помошю производяших функщий. Пусть $\xi(z)=\sum_{i=1}^{\infty} z^{i} / i$; заметим, что $\xi(z)=-\log (1-z)$ при $|z|<1$, и, кроме того,

$$
(\xi(z))^{m}=\sum_{j=m}^{\infty} z^{j}\left\{\sum_{\substack{i_{1}+\cdots+i_{m}=j \\ i_{1}, \ldots, i_{m}>0}} \frac{1}{i_{1} \cdots i_{m}}\right\}=(-\log (1-z))^{m} .
$$

С учетом соотношения

$$
\left.\frac{1}{N !} \frac{d^{N}}{d z^{N}}\left\{\left(\log \frac{1}{1-z}\right)^{m}\right\}\right|_{z=0}=\sum_{\substack{n_{1}+\cdots+n_{m}=N \\ n_{1}, \ldots, n_{m}>0}} \frac{1}{n_{1} \cdots n_{m}}
$$

мы получаем второе выражение в (1). Это завершает доказательство.

Любопытно отметить, что $s(N, m)=\left.\frac{N !}{m !} \frac{d^{N}}{d z^{N}}\left\{\left(\log \frac{1}{1-z}\right)^{m}\right\}\right|_{z=0}$ - это числа Стирлинга первого рода, изученные в теории чисел и комбинаторике. Голди отметил этот факт в своем обзоре [8].

Эта теорема позволяет передоказать результат п. 2.2.

СлЕДСТВИЕ 2.4.1. Математическое ожидание числа отрезков, составляющих выпуклую миноранту, отвечающую случайному блужданию длины $N$, равно $\sum_{m=1}^{N} 1 / m$. Дисперсия числа отрезков равна $\sum_{m=1}^{N}\left(1 / m-1 / m^{2}\right)$.

ДокАЗАТЕльство. Непосредственно вычисляем:

$$
\begin{aligned}
& \left.\sum_{m=1}^{N} m \frac{1}{m ! N !} \frac{d^{N}}{d z^{N}}\left\{\left(\log \frac{1}{1-z}\right)^{m}\right\}\right|_{z=0} \\
& \quad=\left.\frac{1}{N !} \frac{d^{N}}{d z^{N}}\left\{\left(\log \frac{1}{1-z}\right) \sum_{m=1}^{N} \frac{1}{(m-1) !}\left(\log \frac{1}{1-z}\right)^{m-1}\right\}\right|_{z=0}
\end{aligned}
$$




$$
\begin{aligned}
& =\left.\frac{1}{N !} \frac{d^{N}}{d z^{N}}\left\{\left(\log \frac{1}{1-z}\right)\left(\frac{1}{1-z}-\sum_{m=N}^{\infty} \frac{1}{m !}\left(\log \frac{1}{1-z}\right)^{m}\right)\right\}\right|_{z=0} \\
& =\left.\frac{1}{N !} \frac{d^{N}}{d z^{N}}\left\{\frac{1}{1-z} \log \frac{1}{1-z}\right\}\right|_{z=0} \\
& =\frac{1}{N !} \sum_{m=1}^{N}(m-1) !(N-m) !\left(\begin{array}{l}
N \\
m
\end{array}\right)=\sum_{m=1}^{N} \frac{1}{m}
\end{aligned}
$$

Аналогичное, но более длинное вычисление, дает формулу для дисперсии.

Таким образом, математическое ожидание числа отрезков, составляюших вьпуклую миноранту случайного блуждания длины $N$, растет как $\log N$.

\section{3. Динамика с прилипанием и случайные блуждания}

3.1. Введение. Модель гравитационно взаимодействующих частиц с прилипанием была вначале предложена для изучения задачи о структурах больших масштабов во вселенной [16]. Эта задача привлекла внимание в [7] и [10]; имеется и полезная связь с турбулентностью Бюргерса [1]. Изучаемая нами формулировка задачи восходит к [10] и является упрощенным одномерным вариантом модели Зельдовича; мы обсуждаем также некоторые свойства модели без гравитации.

Рассмотрим систему $N$ точечных масс (частищ) на числовой прямой, притягиваюших друг друга с силами, пропорщиональньми произведениям соответствуюших масс и не зависяшими от расстояний между частищами. Соответствующий $N$-частичный гамильтониан имеет вид

$$
H_{N}=\sum_{i=1}^{N} \frac{p_{i}^{2}}{2 m_{i}}+\gamma \sum_{i<j} m_{i} m_{j}\left|x_{i}-x_{j}\right|
$$

где $\gamma$ - гравитационная постоянная, $m_{i}, x_{i}$ и $p_{i}$ - масса, положение и импульс частицы $i$, а потенциал - это стандартный одномерньй гравитационньй потенциал. Этот гамильтониан управляет динамикой системы частиц на временны́х интервалах между попарными столкновениями. В нашем случае столкновения совершенно неупруги, т.е. импульс сохраняется, а энергия - нет. Для большей наглядности: при столкновении двух частиц создается бо́льшая частица, масса которой равна сумме масс исходных столкнувшихся частиц, а импульс этой новой частищы равен сумме импульсов исходных частищ. Последуюшая динамика управляется гамильтонианом приведенного выше вида, но с другими $m_{i}, x_{i}$ и $p_{i}$. Это полностью задает правила, определяюшие динамику.

Интересная задача, впервые изученная в [10], может быть описана следующим образом. Пусть $M$ - полная масса введенной выше системы частиц. В начальньй момент времени, $t=0$, имеется $N$ одинаковых частиц, каждая из которых имеет массу $m=M / N$; частицы равномерно распределены на отрезке $[0,1] \subset \mathbb{R}$ в том смысле, что находятся на одинаковом расстоянии $a=1 / N$ друг от друга. Нам нужно следующее полезное обозначение. Рассмотрим кластер размера $n$, начинающийся в частице с номером $j+1$ (при $j+n \leqslant N)$. Обозначим через $X_{j+1}^{n}(t)$ центр масс этого кластера 
частиц $(j+1, \ldots, j+n)$ в момент времени $t$ (перед любым столкновением какой-либо частицы этого кластера с частицей вне кластера); тогда $X_{j+1}^{n}(t)$ равен

$$
X_{j+1}^{n}(t)=\left(j+\frac{n+1}{2}\right) a+\frac{t}{n} \sum_{s=1}^{n} v_{j+s}+\gamma m(N-n-2 j) \frac{t^{2}}{2}
$$

где $v_{i}$ - начальная скорость частицы $i$. В [5] отмечено (см. также [7]), что необходимые и достаточные условия образования агрегата массы $m n$ за время $t$ из кластера $(j+1, \ldots, j+n)$, подчиненного ограничению, что все частицы, не принадлежашие этому кластеру, остаются на конечном расстоянии от полученного агрегата по крайней мере вплоть до момента $t$ включительно, имеют следующий вид:

$$
\begin{aligned}
X_{j+1}^{r}(t) & >X_{j+r+1}^{n-r}(t), & r & =1,2, \ldots, n-1, \\
X_{j-s+1}^{s}(t) & <X_{j+1}^{n}(t), & s & =1,2, \ldots, j, \\
X_{j+1}^{n}(t) & <X_{j+n+1}^{s}(t), & s & =1,2, \ldots, N-(n+j) .
\end{aligned}
$$

Последнее условие содержательно только при $j<N-n$. Первое условие утверждает, что для любого разбиения набора $(j+1, \ldots, j+n)$ на левую и правую часть центры масс этих двух кусков должны пересечься к моменту времени $t$. Второе и третье условия просто означают тот факт, что кластер $(j+1, \ldots, j+n)$ не взаимодействует с частицами, ему не принадлежашими.

Обозначим через $\Theta(x)$ функцию Хевисайда, принимающую значение 1 при $x>0$ и 0 в противном случае. Непосредственная подстановка соотношения (4) в вьшеприведенные условия позволяет нам переписать их в виде

$$
\begin{array}{r}
\prod_{r=1}^{n-1} \Theta\left(\sum_{l=1}^{r} v_{j+l}-\frac{r}{n} \sum_{l=1}^{n} v_{j+l}+\frac{m \tau r(n-r)}{2}\right)=1, \\
\prod_{r=0}^{j-1} \Theta\left(\frac{j-r}{(j-r)+n} \sum_{s=1}^{(j-r)+n} v_{r+s}-\sum_{s=1}^{j-r} v_{r+s}-\frac{m n(j-r) \tau}{2}\right)=1, \\
\prod_{s=1}^{N-(j+n)} \Theta\left(\frac{n}{n+s} \sum_{i=1}^{n+s} v_{j+i}-\sum_{i=1}^{n} v_{j+i}-\frac{m n s \tau}{2}\right)=1,
\end{array}
$$

где $\tau=\gamma t-1 /(\rho t)$ и $\rho=m / a$.

Пусть $v_{1}, \ldots, v_{N}$ - вешественные независимые одинаково распределенные случайные величины, имеюшие плотности, которые симметричны относительно 0 ; мы предположим также, что случайные величины имеют моменты порядка $4+\delta$ для некоторого $\delta>0$. Мы изучаем следуюшие вопросы: (i) какие вероятностные утверждения могут быть высказаны об агрегационном процессе для динамики с гравитацией? (ii) какие утверждения такого типа могут быть высказаны об агрегационном процессе для динамики без гравитации? В [5] задача (i) изучена в случае, когда начальное распределение скоростей является гауссовым. Авторы обнаружили критический момент времени $t^{*}$ и показали, что до момента $t^{*}$ агрегатов макроскопической массы нет, 
а после этого момента макроскопическая масса есть. Использованные в [5] методы включают явные вычисления с гауссовыми распределениями и теорему Спарре Андерсена. В [5] также высказана гипотеза, что до наступления критического момента могут образовьваться только кластеры размера $\sqrt{N}$.

В задаче (i) мы определяем такой же критический момент времени $t^{*}$, до которого вероятность наличия массивного агрегата равна 0 , а после -1 ; мы назовем $t^{*}$ моментом скачка и отметим, что $t^{*}$ зависит только от $\gamma$ и $\rho$ (в частности, $t^{*}=1 / \sqrt{\gamma \rho}$, что соответствует $\tau=0$ ). Мы полностью описываем агрегационньй процесс в критический момент времени и показываем, что его статистика существенно отличается от упомянутого ранее режима. Эти утверждения должны пониматься в непрерывном пределе, где $N \rightarrow \infty$ так, что $M=m N$, а величина $\rho=m / a$ остается постоянной. В случае конечного числа частиц мы приводим явную формулу для вероятности наличия $r$ масс в момент $t^{*}$; отсюда следует очень простая формула для математического ожидания числа масс в момент $t^{*}$. С физической точки зрения важно отметить, что $t^{*}-$ это в точности момент времени, к которому система с любыми начальными скоростями в момент времени 0 коллапсирует, образуя единственный массивньй агрегат; тот факт, что это происходит при больших $N$, показьвает, что динамика, хотя и случайная, на самом деле управляется гравитационными влияниями. Для лучшего понимания предельного процесса мы найдем последовательность моментов времени $\tau_{N}$, для которых вьшеприведенные вероятности допускают равномерньй контроль. Мы также находим частичное подтверждение упомянутой вьше гипотезы Мартена-Пясецки [10]. В задаче (ii) мы вычисляем вероятность наличия $r$ масс при данных $N$ начальных массах в пределе при $t \rightarrow \infty$; при этом в баллистическом случае $(\gamma=0)$ динамические свойства существенно отличаются от гравитационного случая.

3.2. Отсутствие макроскопических масс при $\tau<0<\gamma$. Зафиксируем $\gamma>0$, $\varepsilon>0$ и $\tau<0$. Вероятность того, что агрегат, масса которого больше $\varepsilon M$, создается за время $\tau$, стремится к 0 в непрерьвном пределе; однако на самом деле утверждение теоремы несколько сильнее. Пусть $\delta$ определено, как во введении. Выберем любое $\nu \in(1-\delta /(2(6+\delta)), 1]$ и определим $A_{\delta}^{N}(\tau)$ как множество начальных скоростей, для которых агрегат, масса которого не меньше $\varepsilon m N^{1 /(2-\nu)}$, создается за время $\tau$; число $N$ назьвается начальным числом частии в системе.

Teopema 3.2.1. $\mathrm{P}\left(A_{\delta}^{N}(\tau)\right) \rightarrow 0$ npu $N \rightarrow \infty$.

Перед началом вычислений заметим, что часто будут появляться бессмысленные выражения вроде $n / 2$ (где $n$ не обязательно четно); они помогают избежать педантичных выражений вроде "наибольшего целого, не превосходящего . . . "; бывает, что эти величины сушественны, но в этом разделе они не играют роли и будут игнорироваться. Мы сначала оценим родственную величину, которую мы обозначим через $p_{N}^{n}(\tau)$ и определим формулой

$$
\mathrm{P}\left(\prod_{r=1}^{n-1} \Theta\left(X_{1}^{r}(t)-X_{r+1}^{n-r}(t)\right)=1\right)=\mathrm{P}\left(\prod_{r=1}^{n-1} \Theta\left(\sum_{l=1}^{r} v_{l}-\frac{r}{n} \sum_{l=1}^{n} v_{l}+\frac{m \tau r(n-r)}{2}\right)=1\right)
$$


очевидно, что $p_{N}^{n}(\tau)$ ограничена величиной

$$
\mathrm{P}\left(\sum_{l=1}^{n / 2} v_{l}-\frac{1}{2} \sum_{l=1}^{n} v_{l}+\frac{m \tau n^{2}}{8}>0\right)
$$

При $n>(\varepsilon N)^{1 /(2-\nu)}$ мы можем продолжить оценку сверху с помошњю величины

$$
\begin{aligned}
\mathrm{P}\left(\sum_{l=1}^{n / 2} v_{l}-\frac{1}{2} \sum_{l=1}^{n} v_{l}+\frac{\varepsilon M \tau n^{\nu}}{8}>0\right) & \leqslant 2 \mathrm{P}\left(\left|\sum_{l=1}^{n} v_{l}\right|>\left(-\frac{\varepsilon M \tau}{16}\right) n^{\nu}\right) \\
& \leqslant \mathscr{C}\left(-\frac{16}{\varepsilon M \tau}\right)^{4+\delta} \frac{1}{n^{(4+\delta)(\nu-1 / 2)}} \\
& \leqslant \mathscr{D}\left(-\frac{16}{\varepsilon M \tau}\right)^{4+\delta} \frac{1}{N^{\frac{(4+\delta)(\nu-1 / 2)}{2-\nu}}}
\end{aligned}
$$

где $\mathscr{C}$ - постоянная, зависящая только от моментов случайных величин $v_{i}$, а $\mathscr{D}$ - постоянная, включающая некоторьй множитель с $\varepsilon$. Последняя оценка является комбинацией неравенства Чебышева, неравенства Марцинкевича-Зигмунда и небольшого рассуждения; неравенство Марцинкевича-Зигмунда и упомянутое рассуждение приведены в приложении. Эти неравенства нужны в доказательстве соотношения

$$
\mathrm{E}\left|\sum_{i=1}^{n} v_{i}\right|^{4+\delta}=O\left(n^{2+\delta / 2}\right)
$$

Вероятность того, что агрегат, масса которого $m n>m(\varepsilon N)^{1 /(2-\nu)}$, образуется к моменту времени $\tau$, может быть очевидньм образом оценена величиной

$$
\sum_{j=1}^{N-n} p_{N}^{n}(\tau)=(N-n) p_{N}^{n}(\tau)
$$

Эта оценка отражает тот факт, что $n$-кластер может начинаться с любой из первых $N-n$ частищ. Суммируя по $n>(\varepsilon N)^{1 /(2-\nu)}$, получаем:

$$
\sum_{n>(\varepsilon N)^{1 /(2-\nu)}}(N-n) p_{N}^{n}(\tau) \leqslant N^{2} p_{N}^{(\varepsilon N)^{1 /(2-\nu)}}(\tau) \leqslant \frac{\mathscr{D}(\varepsilon)}{N^{\frac{(\nu-1 / 2)(4+\delta)}{2-\nu}-2}}\left(-\frac{16}{\varepsilon M \tau}\right)^{4+\delta}
$$

Это последнее выражение стремится к нулю при $N \rightarrow \infty$. Таким образом, вероятность иметь массивньй агрегат, являющийся конечной долей полной массы $M$, к моменту времени $\tau<0$ стремится к нулю при $N \rightarrow \infty$; это утверждение дает также некоторую информацию о несушествовании агрегатов меньших масс. Заметим, что мы не использовали существования плотностей у величин $v_{1}, \ldots, v_{N}$. 
3.3. Агрегация масс в критический момент времени $\tau=0$. Пусть $N$ - начальное число частиц. Пусть $V_{r}^{N}$ - множество таких начальных скоростей $\left(v_{1}, \ldots, v_{N}\right)$ $\in \mathbb{R}^{N}$, что к моменту $\tau=0$ имеется в точности $r$ масс. Определим $W_{n}^{N}$ как множество таких начальных скоростей $\left(v_{1}, \ldots, v_{N}\right) \in \mathbb{R}^{N}$, что к моменту времени $\tau=0$ имеется агрегат с массой, равной в точности $M n / N$.

TeOpema 3.3.1. $\mathrm{P}\left(V_{r}^{N}\right)=\left.\frac{1}{r ! N !} \frac{d^{N}}{d z^{N}}\left\{\left(\log \frac{1}{1-z}\right)^{r}\right\}\right|_{z=0}$.

Teоpema 3.3.2. $\mathrm{P}\left(W_{n}^{N}\right)=\frac{1}{n}$ npu $n>\frac{N}{2}$.

СлЕДСтвиЕ 3.3.1. Математическое ожидание числа масс к моменту времени $t^{*}$ равно $\sum_{j=1}^{N} \frac{1}{j}$.

Заметим сначала, что случай $\tau=0$ имеет смысл только при $\gamma>0$. Мы увидим, что ключ к пониманию статистики агрегационного процесса лежит в понимании статистических свойств выпуклой миноранты процесса симметрического случайного блуждания, порожденного вешественными независимьми одинаково распределенными случайными величинами $v_{1}, \ldots, v_{N}$. В частности, при $\tau=0$ соотношения (5), (6) и (7) дают точное соотношение между скоростями $v_{1}, \ldots, v_{N}$ и вьпуклой минорантой функции, начинаюшейся в нуле и имеющей приращения $v_{1}, \ldots, v_{N}$ (для блуждания, порожденного $\left.v_{1}, \ldots, v_{N}\right)$. Мы можем извлечь отсюда следующую информацию: (i) число отрезков в вьпуклой миноранте в точности равно числу агрегатов к моменту времени $\tau=0$ при данных начальных скоростях $v_{1}, \ldots, v_{N}$; (ii) массы этих агрегатов - это просто длины соответствуюших отрезков, умноженные на массу $m$ начальных точечных масс; (iii) порядок следования этих масс совпадает с порядком следования длин отрезков.

Это замечательное соответствие позволяет вычислить статистические свойства агрегации масс в момент времени $\tau=0$, вычисляя статистические свойства вьпуклой миноранты процесса случайного блуждания, порожденного симметричными независимыми одинаково распределенными случайньми величинами $v_{1}, \ldots, v_{N}$. Следуюшие статистические свойства этого объекта были изучены в разделе 2: (i) при $N / 2<n \leqslant N$ вероятность получить отрезок длины $n$ в вьпуклой миноранте равна $1 / n$, поэтому, если $1 / 2<s<t \leqslant 1$, то вероятность получить агрегат массы $\mathscr{M}$ с $s M \leqslant \mathscr{M} \leqslant t M$ равна сумме $\sum_{s N \leqslant n \leqslant t N} 1 / n$, которая стремится к $\log (t / s)$ при $N \rightarrow \infty$ (это совершенно другая величина, чем в случае $\tau<0$ ); (ii) при данных $N$ начальных точечных массах вероятность того, что при $\tau=0$ имеется в точности $r$ агрегатов, равна

$$
\frac{1}{r !} \sum_{\substack{n_{1}+\cdots+n_{r}=N \\ n_{1}, \ldots, n_{r}>0}} \frac{1}{n_{1} \cdots n_{r}} .
$$

Используя метод порождаюших функций, нетрудно показать, что это выражение равно

$$
\left.\frac{1}{r ! N !} \frac{d^{N}}{d z^{N}}\left\{\left(\log \frac{1}{1-z}\right)^{r}\right\}\right|_{z=0} .
$$


Заметим, что в этих утверждениях мы не используем условия существования моментов порядка $4+\delta$.

Далее, мы можем изучить агрегационньй процесс в непрерьвном пределе в критический момент времени $\tau=0$. В соответствии со сделанньми вьше замечаниями, эта задача в точности совпадает со статистической задачей о длинах отрезков в вьпуклой миноранте броуновского движения на $[0,1]$; мы подробно изучили эту задачу в разделе 2 . В частности, вероятностное распределение наибольшей массы в точности соответствует вероятностному распределению длины наибольшего отрезкавыпуклой миноранты броуновского движения на $[0,1]$; вероятностное распределение второй по величине массы в точности соответствует вероятностному распределению второго по длине отрезка выпуклой миноранты броуновского движения на $[0,1]$, и т.д. Это соответствие позволяет установить следующий факт о системе в непрерывном пределе.

ТЕОРема 3.3.3. Функиия распределения для $r$-й по величине массы равна $F_{r}(\xi / M)$, где $F_{r}(\xi)$ определена в разделе 2.

Теорема 3.3.4. Плотность совместного распределения $r$ наибольших масс равна $p_{r}\left(x_{1} / M, \ldots, x_{r} / M\right)$ на $\Lambda_{r}$, әде $F_{r}(\xi)$ и $p_{r}\left(x_{1}, \ldots, x_{r}\right)$ определены в разделе 2.

3.4. Агрегат полной массы при $\tau>0$. Зафиксируем $\rho \in(1-\delta /(2(4+\delta)), 1]$, $\tau, \varepsilon>0$. Пусть дано $N$ начальных частиц, и пусть $L_{\rho}^{N}-$ множество таких начальных скоростей $\left(v_{1}, \ldots, v_{N}\right) \in \mathbb{R}^{N}$, что в момент времени $\tau$ имеется агрегат массы $M n / N$ при некотором $n$, удовлетворяюшем условию $\varepsilon N^{\rho} \leqslant n \leqslant(1-\varepsilon) N$. Вот основной результат этого пункта.

TEOPEMA 3.4.1. $\mathrm{P}\left(L_{\rho}^{N}\right) \rightarrow 0$ nрu $N \rightarrow \infty$.

Вероятность того, что есть агрегат, составленный в точности из $n$ начальных частиц, ограничена величиной

$$
\begin{aligned}
\sum_{j=0}^{N-n} \mathrm{P} & \left(\prod_{r=0}^{j-1} \Theta\left(X_{j+1}^{n}(t)-X_{r+1}^{j-r}(t)\right)=1\right) \\
& \times \mathrm{P}\left(\prod_{r=1}^{n-1} \Theta\left(X_{j+1}^{r}(t)-X_{j+r+1}^{n-r}(t)\right)=1\right) \\
& \times \mathrm{P}\left(\prod_{s=1}^{N-(j+n)} \Theta\left(X_{j+n+1}^{s}(t)-X_{j+1}^{n}(t)\right)=1\right) \\
\leqslant & \sum_{j=0}^{N-n} \mathrm{P}\left(\prod_{r=0}^{j-1} \Theta\left(X_{j+1}^{n}(t)-X_{r+1}^{j-r}(t)\right)=1\right) \\
& \times \mathrm{P}\left(\prod_{s=1}^{N-(j+n)} \Theta\left(X_{j+n+1}^{s}(t)-X_{j+1}^{n}(t)\right)=1\right)
\end{aligned}
$$




$$
\begin{aligned}
\leqslant & \left\{\sum_{j=0}^{(N-n) / 2}+\sum_{j=(N-n) / 2}^{N-n}\right\} \\
& \mathrm{P}\left(\prod_{r=0}^{j-1} \Theta\left(\frac{j-r}{(j-r)+n} \sum_{s=1}^{(j-r)+n} v_{r+s}-\sum_{s=1}^{j-r} v_{r+s}-\frac{m n(j-r) \tau}{2}\right)=1\right) \\
& \times \mathrm{P}\left(\prod_{s=1}^{N-(j+n)} \Theta\left(\frac{n}{n+s} \sum_{i=1}^{n+s} v_{j+i}-\sum_{i=1}^{n} v_{j+i}-\frac{m n s \tau}{2}\right)=1\right) .
\end{aligned}
$$

Положим $\Sigma_{1}=\sum_{j=0}^{(N-n) / 2}$ и $\Sigma_{2}=\sum_{j=(N-n) / 2}^{N-n}$ в предыдущей формуле. Мы можем следуюшим образом оценить эти величины:

$$
\begin{aligned}
& \Sigma_{1} \leqslant(N-n) \mathrm{P}\left(\Theta\left(\frac{1}{n+\frac{N-n}{2}} \sum_{i=1}^{n+\frac{N-n}{2}} v_{i}-\frac{1}{n} \sum_{i=1}^{n} v_{i}-\frac{m \tau}{2} \frac{N-n}{2}\right)=1\right), \\
& \Sigma_{2} \leqslant(N-n) \mathrm{P}\left(\Theta\left(\frac{1}{n+\frac{N-n}{2}} \sum_{s=1}^{n+\frac{N-n}{2}} v_{s}-\frac{2}{N-n} \sum_{s=1}^{\frac{N-n}{2}} v_{s}-\frac{m n \tau}{2}\right)=1\right) .
\end{aligned}
$$

Оценки для (11) и (12) похожи, и мы ограничимся кратким обсуждением (11). Мы оцениваем первую вероятность в $\Sigma_{1}$ единицей, а вторую - сознавая, что $N-(j+n)>$ $(N-n) / 2$ в области изменения $j$ в сумме $\Sigma_{1}$ и что $v_{1}, \ldots, v_{N}$ - независимые одинаково распределенные случайные величины; этот факт позволяет подобрать наиболее сильную оценку, обшую для всех членов в $\Sigma_{1}$, и мажорировать $\Sigma_{1}$ произведением этой оценки на $N-n$. Оценка в (12) проводится аналогично и с той лишь разницей, что единицей оценивается вторая вероятность. Суммируя по всем таким $n$, что $\varepsilon N^{\rho} \leqslant n \leqslant(1-\varepsilon) N$, где $\rho \in(1-\delta /(2(4+\delta)), 1]$, мы получаем оценку

$$
\sum_{\varepsilon N^{\rho} \leqslant n \leqslant(1-\varepsilon) N}\left\{\Sigma_{1}+\Sigma_{2}\right\} \leqslant 4 N^{2} \mathrm{P}\left(\frac{1}{N} \sum_{i=1}^{N} v_{i} \geqslant \frac{M \tau}{4 N^{1-\rho}}\right) .
$$

Используя оценки, аналогичные полученным в разделе 2 , и наличие моментов порядка $4+\delta$, видим, что последнее выражение стремится к нулю при $N \rightarrow \infty$. В силу результатов п. 3.3 отсюда следует, что для любого $\tau>0$ вероятность наличия агрегата полной массы стремится к 1 в непрерывном пределе. Важно отметить, что остаточная пыль частиц должна быть пренебрежимой в непрерьвном пределе. Заметим также, что в этом рассуждении мы не нуждаемся в предположении, что распределения скоростей имеют плотности.

3.5. Равномерный контроль предельного процесса: последовательность моментов времени. Зафиксируем $\varepsilon, \xi>0$. Мы сейчас построим последовательность положительных чисел $\tau_{N}$, стремящуюся к 0 и такую, что вероятность иметь агрегат, составленный из $n>(1-\varepsilon) N$ частиц из $N$ начальных частиц, больше величины $1-\xi$; это есть вероятность получить агрегат массы $\mathscr{M}>(1-\varepsilon) M$ к моменту времени $\tau_{N}$ (где $N$ - начальное число частиц), большая, чем $1-\xi$. 
Рассмотрим последовательность $\tau_{N}=\mathscr{D} N^{-\frac{1}{2}\left(\rho-\left(1-\frac{\delta}{2(4+\delta)}\right)\right)} ;$ при подходяшем выборе $\mathscr{D}$ рассматриваемая вероятность ограничена снизу числом $1-\xi$ равномерно по $N$. Чтобы убедиться в этом, мы должны только заменить $\tau$ на $\tau_{N}$ в выражении (13). Оценим эту новую величину с помошю неравенства Чебьшева и упомянутых вьше фактов, связанных с моментами случайных величин $\left|\sum_{i=1}^{n} v_{i}\right|$ при $n \rightarrow \infty$ :

$$
\begin{aligned}
4 N^{2} \mathrm{P}\left(\frac{1}{N} \sum_{i=1}^{N} v_{i} \geqslant \frac{M \tau_{N}}{4 N^{1-\rho}}\right) & \leqslant \frac{4 N^{2} \mathscr{R} N^{2+\delta / 2}}{(\mathscr{D} N)^{\frac{1}{2}(\rho+1)(4+\delta)}} \\
& =\frac{4 \mathscr{R}}{\mathscr{D}^{\frac{1}{2}(4+\delta)(\rho+1)} N^{\frac{1}{2}(4+\delta)(\rho+1)-(4+\delta / 2)}}
\end{aligned}
$$

где $\infty>\mathscr{R}>\lim \sup \mathrm{E}\left|\sum_{i=1}^{n} v_{i}\right|^{4+\delta} / n^{2+\delta / 2}$. Таким образом, беря $\mathscr{D}$ достаточно большим, мы можем оценить эту вероятность равномерно по $N$ со сколь угодно точным контролем; в частности, мы можем оценить эту вероятность величиной $\xi / 2$, что показывает, что наш выбор $\tau_{N}$ удовлетворителен.

3.6. Баллистическая агрегация: $\gamma=0$. В этом пункте мы рассмотрим баллистический случай, в котором $\gamma=0$; это - случай отсутствия гравитащии. В этом режиме $\tau=-1 /(\rho t)$ при $t>0$, и $N$ обозначает начальное число частиц. Пусть $\Omega_{N}^{r}-$ множество таких начальных скоростей $v_{1}, \ldots, v_{N}$, что конечное число агрегатов (после завершения агрегационного процесса) равно $r$. Из условий (5), (6) и (7) следует, что

$$
\mathrm{P}\left(\Omega_{N}^{r}\right)=\frac{1}{r !} \sum_{\substack{n_{1}+\cdots+n_{r}=N \\ n_{1}, \ldots, n_{r}>0}} \frac{1}{n_{1} \cdots n_{r}}
$$

Эту формулу можно получить теми же методами, что и в п. 3.3 ; соответствие с выпуклой минорантой отличается только в том, что вьпуклая миноранта дает поведение на больших временах, в отличие от поведения вблизи $\tau=0$. Это показывает, что динамические свойства баллистического случая существенно отличаются от динамических свойств гравитационного случая. Как и в разделе 2 , заметим, что математическое ожидание числа масс равно $\sum_{j=1}^{N} 1 / j ;$ при $N \rightarrow \infty$ число масс растет как $\log N$.

\section{4. Заключительные замечания}

В разделе 2 мы доказали некоторые утверждения о выпуклых минорантах случайных блужданий, порожденных независимыми одинаково распределенными случайными величинами с непрерьвными распределениями. Бо́льшая часть этих фактов связана с применением свойств циклических сдвигов, описанных в разделе 2. Единственным дополнительным инструментом является разложение $\mathbb{R}^{n}$ на части, которые тонко связаны с путями случайного блуждания. Мы полностью охарактеризовали длину $r$-го по длине отрезка вьпуклой миноранты броуновского движения на конечных временны́х интервалах, а также совместные плотности набора $r$ наиболее длинных отрезков; асимптотические теоремы теории случайных перестановок играют здесь решающую роль. 
В разделе 3 рассмотрены некоторые вопросы об одномерном гравитационно взаимодействующем газе со случайными начальными скоростями. Мы видели, что в гравитационном случае имеется такой неслучайньй момент времени $t^{*}$, что после этого момента вероятность получить массивный агрегат полной массы стремится к 1 в непрерьвном пределе; до этого момента вероятность получить агрегат, масса которого является конечной долей полной массы системы, стремится к 0 в непрерьвном пределе. Мы называем этот момент времени $t^{*}$ моментом скачка и характеризуем агрегационньй процесс в этот момент, используя результаты раздела 2. Мы также нашли последовательность моментов времени, бо́льших, чем $t^{*}$ и стремяшихся к нему, таких, что контроль указанных выше вероятностей равномерен при увеличении числа частищ. Этот анализ приводит к вьводу, что в гравитационном случае динамика управляется гравитацией и по существу неслучайна. Нами рассмотрен также баллистический случай, в котором $\gamma=0$. Мы исследовали долгосрочное поведение для системы $N$ частиц со случайными начальньми скоростями и заключили, что это поведение значительно отличается от поведения в гравитационном случае.

\section{5. Приложение}

5.1. Некоторые теоремы о конечных последовательностях вещественных чисел. Начнем с определения некоторых понятий. Пусть $T: \mathbb{R}^{n} \rightarrow \mathbb{R}^{n}$ - оператор циклического сдвига, $T\left(x_{1}, \ldots, x_{n}\right)=\left(x_{2}, \ldots, x_{n}, x_{1}\right)$. Заметим, что $T$ - биекция, и введем обозначение $T^{m}=T \circ \cdots \circ T$ ( $m$ сомножителей $)$. При $a=\left(a_{1}, \ldots, a_{n}\right) \in \mathbb{R}^{n}$ определим блуждание $R_{t}(a)$, порожденное $a$, следующим образом: $R_{0}(a)=0, R_{t}(a)=\sum_{i=1}^{t} a_{i}$ при $t \in\{1, \ldots, n\}$ и, наконец, если $t \in(0, n)$, то $R_{t}(a)=R_{\lfloor t\rfloor}(a)+(t-\lfloor t\rfloor) a_{\lfloor t\rfloor+1}$, где $\lfloor t\rfloor$ означает наибольшее целое число, меньшее $t$. Определим $m$-й циклический сдвиг блуждания $R_{t}(a)$, порожденного $a \in \mathbb{R}^{n}$, как блуждание $R_{t}\left(T^{m}(a)\right)$. Пусть $\mu$ - положительная $T$-инвариантная борелевская мера на $\mathbb{R}^{n}$, абсолютно непрерывная относительно меры Лебега, и при $r>0$ определим положительную борелевскую меру $\mu^{r}$ формулой $\mu^{r}(C)=\mu\left(C \cap B_{r}\right)$, где $B_{r}$ - открытьй шар радиуса $r$ с центром в начале координат и $C$ - произвольное борелевское множество; заметим, что $\mu^{r}$ тоже $T$-инвариантна. Положим $A=\left\{a=\left(a_{1}, \ldots, a_{n}\right) \in \mathbb{R}^{n}: R_{t}(a)>(t / n) R_{n}(a), \forall t \in(0, n)\right\}$. Пусть $\mathscr{B}_{\alpha}=\left\{a=\left(a_{1}, \ldots a_{n}\right) \in \mathbb{R}^{n}: \sum_{i=1}^{n} a_{i}=\alpha\right\}$ при $\alpha \in \mathbb{R}$. Сформулируем две леммы, доказательство которых отложим до конца этого пункта, и затем приведем некоторые теоремы и их вероятностные следствия.

ЛЕмма 5.1.1. Если $0 \leqslant j \neq l<n, \operatorname{mo} T^{j}(A) \cap T^{l}(A)=\varnothing$.

ЛЕмма 5.1.2. $\mu\left\{\left(\bigcup_{i=0}^{n-1} T^{i}(A)\right)^{c}\right\}=0$.

Теорема 5.1.1. При $\infty>r>0$ мера $\mu^{r}$ конечна. Кроме того,

$$
\frac{\mu^{r}(A)}{\mu^{r}\left(\mathbb{R}^{n}\right)}=\frac{1}{n}, \quad u \quad \lim _{r \rightarrow \infty} \frac{\mu^{r}(A)}{\mu^{r}\left(\mathbb{R}^{n}\right)}=\frac{1}{n} .
$$

В частности, если $\mu$ - вероятностная мера, то $\mu(A)=1 / n$. 
ДоКАЗАТЕЛЬСТВо. Это - простое следствие лемм 5.5.1 и 5.1.2 и того факта, что мера $\mu T$-инвариантна.

СлЕДСТВИЕ 5.1.1. Если $X_{1}, \ldots, X_{n}$ - независимые одинаково распределенные вещественные случайные величины с плотностями, то

$$
\mathrm{P}\left(\sum_{j=1}^{i} X_{j}>\frac{i}{n} \sum_{j=1}^{n} X_{j}, \forall i \in\{1, \ldots, n-1\}\right)=\frac{1}{n},
$$

где $\mathrm{P}$ - совместная вероятностная мера, порожденная $X_{1}, \ldots, X_{n}$.

Заметим, что мы не предполагаем существования никаких моментов.

ДокАЗАТЕльство. Достаточно проверить, что мера-произведение на $\mathbb{R}^{n}$, порожденная плотностями величин $X_{1}, \ldots, X_{n}$, удовлетворяет приведенным вьше требованиям к $\mu$.

Пусть $g \in L_{\text {loc }}^{1}\left(\mathbb{R}^{n}\right)$ - плотность меры $\mu$ относительно лебеговой меры. Определим существенньй носитель $\operatorname{ess}(\mu)$ меры $\mu$ следующим образом. Пусть $\Omega$ - семейство таких открытых множеств $\omega \subset \mathbb{R}^{n}$, что $g(x)=0$ для $\mu$-почти всех $x \in \omega$. Пусть $\omega^{*}=\bigcup_{\omega \in \Omega} \omega$; тогда $\operatorname{ess}(\mu)=\left(\omega^{*}\right)^{c}$. Пусть $\mathscr{A}_{\mu}-$ множество всех таких $\alpha \in \mathbb{R}$, что $\operatorname{ess}(\mu) \cap \mathscr{B}_{\alpha} \cap(\partial(\operatorname{ess}(\mu)))^{c} \neq \varnothing$. Для $\alpha \notin \mathscr{A}_{\mu}$ положим $\mu\left(A \mid \mathscr{B}_{\alpha}\right)=0$, и при $\alpha \in \mathscr{A}_{\mu}$ введем величину $\mu\left(A \mid \mathscr{B}_{\alpha}\right)=\lim _{\varepsilon \rightarrow 0} \mu_{\varepsilon}\left(A \mid \mathscr{B}_{\alpha}\right)$, где

$\mu_{\varepsilon}\left(A \mid \mathscr{B}_{\alpha}\right)=\lim _{r \rightarrow \infty} \frac{\mu^{r}\left(A \cap \mathscr{B}_{\alpha}^{\varepsilon}\right)}{\mu^{r}\left(\mathscr{B}_{\alpha}^{\varepsilon}\right)}, \quad \mathscr{B}_{\alpha}^{\varepsilon}=\left\{a=\left(a_{1}, \ldots, a_{n}\right) \in \mathbb{R}^{n}:\left|\alpha-\sum_{i=1}^{n} a_{i}\right|<\varepsilon\right\}$.

Как мы увидим в доказательстве следуюшей теоремы, эти пределы существуют почти всюду.

Tеорема 5.1.2. Если $\alpha \in \mathscr{A}_{\mu}, \operatorname{mo} \mu\left(A \mid \mathscr{B}_{\alpha}\right)=1 / n$.

ДокАЗАТЕЛЬСТВо. Начнем со следуюших наблюдений:

(i) $T \mathscr{B}_{\alpha}^{\varepsilon}=\mathscr{B}_{\alpha}^{\varepsilon}$;

(ii) поскольку $x \in B_{r}$ тогда и только тогда, когда $T x \in B_{r}$, и мера $\mu^{r}$ является $T$-инвариантной, то $\mu^{r}\left(T \mathscr{B}_{\alpha}^{\varepsilon}\right)=\mu^{r}\left(\mathscr{B}_{\alpha}^{\varepsilon}\right)$;

(iii) если $0 \leqslant j \neq l<n$, то, по лемме 5.1.1, из соотношений $T^{j}\left(A \cap \mathscr{B}_{\alpha}^{\varepsilon}\right)=T^{j}(A) \cap \mathscr{B}_{\alpha}^{\varepsilon}$ и $T^{l}\left(A \cap \mathscr{B}_{\alpha}^{\varepsilon}\right)=T^{l}(A) \cap \mathscr{B}_{\alpha}^{\varepsilon}$ следует, что $T^{j}\left(A \cap \mathscr{B}_{\alpha}^{\varepsilon}\right) \cap T^{l}\left(A \cap \mathscr{B}_{\alpha}^{\varepsilon}\right)=\varnothing ;$

(iv) так как мера $\mu^{r} T$-инвариантна, то $\mu^{r}\left(T^{j}\left(A \cap \mathscr{B}_{\alpha}^{\varepsilon}\right)\right)=\mu^{r}\left(T^{l}\left(A \cap \mathscr{B}_{\alpha}^{\varepsilon}\right)\right)$;

(v) $\mu^{r}\left(\mathscr{B}_{\alpha}^{\varepsilon} \cap\left(\bigcup_{i=0}^{n-1} T^{i}\left(A \cap \mathscr{B}_{\alpha}^{\varepsilon}\right)\right)^{c}\right) \leqslant \mu^{r}\left(\left(\bigcup_{i=0}^{n-1} T^{i}(A)\right)^{c}\right)=0$ по лемме 5.1.2.

Отсюда следует, что при $\alpha \in \mathscr{A}_{\mu}, \varepsilon>0$ и достаточно большом $r$ имеем $\mu^{r}\left(A \cap \mathscr{B}_{\alpha}^{\varepsilon}\right)=$

$\frac{1}{n} \mu^{r}\left(\mathscr{B}_{\alpha}^{\varepsilon}\right)$. Таким образом, вышеприведенные пределы сушествуют, что доказывает требуемый результат.

СлЕДСТВИЕ 5.1.2. Пусть $X_{1}, \ldots, X_{n}$ - вещественные независимье одинаково распределенные случайные величины, имеющие плотности. Пусть $\mathrm{P}$ - совместное вероятностное распределение, порожденное $X_{1}, \ldots, X_{n}, \mathrm{P}^{\prime}$ - мера на $\mathbb{R}^{n}$, индуцированная Р. Для любого $\alpha \in \mathscr{A}_{\mathrm{P}^{\prime}}$ имеем

$$
\mathrm{P}\left(\sum_{j=1}^{i} X_{j}>\frac{i}{n} \sum_{j=1}^{n} X_{j}, \forall i \in\{1, \ldots, n-1\} \mid \sum_{j=1}^{n} X_{j}=\alpha\right)=\frac{1}{n} .
$$


ДокАЗАТЕльство. Нам нужно только проверить, что индуцированная вероятностная мера $\mathrm{P}^{\prime}$ на $\mathbb{R}^{n}$ удовлетворяет условиям теоремы 5.1.2. Эта проверка может быть произведена непосредственно.

Теорему 5.1.2 следует рассматривать как утверждение об условных мерах для $A$, определенных семейством гиперплоскостей $\mathscr{B}_{\alpha}$.

При $a \in \mathbb{R}^{n}$ функция $R_{t}(a)$ непрерьвна на $[0, n]$. Вьпуклая миноранта для $R_{t}(a)$ определяется, как во введении, и обозначается со $(a)$. Теперь мы можем доказать леммы 5.1.1 и 5.1.2.

ДоКАЗАТЕЛЬСТВО ЛЕММЫ 5.1.1. Положим

$$
\begin{aligned}
E=\{a & =\left(a_{1}, \ldots, a_{n}\right) \in \mathbb{R}^{n}: \exists i \in\{1, \ldots, n-1\}, \\
& \left.\exists m \in\{0,1, \ldots, n-1\}, R_{i}\left(T^{m} a\right)=\frac{i}{n} R_{n}(a)\right\} .
\end{aligned}
$$

Так как $E$ - конечное объединение подмногообразий коразмерности один в $\mathbb{R}^{n}$, то лебегова мера $E$ равна нулю, откуда следует, что $\mu(E)=0$. Полагая $F=E^{c}$, мы сейчас покажем, что $F=\bigcup_{j=0}^{n-1} T^{j} A$ и что для любого $a \in F$ есть только один индекс $j$, $0 \leqslant j<n$, зависяший от $a$, для которого $T^{j} a \in A$. Этот факт, вместе с биективностью $T$, завершит доказательство леммы. При $a \in A$ нижеследующее вычисление показывает, что $T^{m} a \notin A$ при $m=1, \ldots, n-1$ :

$$
\begin{aligned}
\frac{R_{m}(a)}{m}>\frac{R_{n}(a)}{n} & \Leftrightarrow \frac{R_{m}(a)}{n-m}>\frac{m}{n(n-m)} R_{n}(a) \\
& \Leftrightarrow \frac{R_{m}(a)}{n-m}>\left(\frac{1}{n-m}-\frac{1}{n}\right) R_{n}(a) \\
& \Leftrightarrow \frac{1}{n-m}\left(R_{n}(a)-R_{m}(a)\right)<\frac{R_{n}(a)}{n} \\
& \Leftrightarrow \frac{1}{n-m} R_{n-m}\left(T^{m} a\right)<\frac{R_{n}(a)}{n} .
\end{aligned}
$$

Отсюда следует, что при $a \in F$ имеется по крайней мере один индекс $j \in\{0, \ldots, n-1\}$, удовлетворяюший условию $T^{j} a \in A$. Мы должны лишш указать алгоритм нахождения такого индекса. Положим $s(a)=R_{n}(a) / n$ и рассмотрим параметризованное семейство прямых в $\mathbb{R}^{2}$, заданное формулой $y_{c}^{a}(x)=s(a) x+c$. Пусть $\mathscr{C}_{a}-$ множество таких $c \in \mathbb{R}$, что $y_{c}^{a}(x)$ лежит ниже $\operatorname{co}(a)$ при $x \in[0, n)$, и пусть $c^{*}=\sup _{c \in \mathscr{C}_{a}} c$. Так как $a \in F$, то прямая $y_{c^{*}}(x)$ лежит под со $(a)$, за исключением одной точки $t_{a} \in[0, n)$, где она пересекает $\operatorname{co}(a)$, и $t_{a} \in\{0,1, \ldots, n-1\}$, поскольку $R_{t}(a)$ - кусочно линейная функция с целочисленными "угловьми" точками. В такой точке с помошью простых геометрических рассуждений, которые могут быть легко формализованы, можно убедиться, что $T^{t_{a}} a \in A$. Если $t_{a}=0$, то $a \in A$, в чем можно убедиться с помощью рисунка (путь $R_{t}(a)$ нигде не пересекает отрезок прямой, соединяющий начало координат с $\left(n, R_{n}(a)\right) \in \mathbb{R}^{2} ;$ а это в точности совпадает с определением условия, что $\left.a \in A\right)$. Если $t_{a} \neq 0$, то мы разобьем $R_{t}(a)$ на две части точкой $t_{a}$ и будем их называть левой и правой частями. Присоединим правую часть к началу координат и левую часть к концу правой части. Это соответствует построению блуждания $R_{t}\left(T^{t_{a}} a\right)$, порожденного 
$T^{t_{a}} a$, и полученное блуждание имеет свойство не пересекать прямую, соединяющую начало координат с $\left(0, R_{n}(a)\right)$. Таким образом, $T^{t_{a}} a \in A$, что завершает доказательство леммы 5.1.1.

ДокАЗАТЕЛЬСТво ЛЕммы 5.1.2. Определим $E$ так же, как и выше, и напомним, что $\mu(E)=0$. Как мы уже знаем, если $a \in F=E^{c}$, то сушествует индекс $j \in$ $\{0,1, \ldots, n\}$, удовлетворяюший условию $T^{j} a \in A$. Поэтому $\left(\bigcup_{j=0}^{n-1} T^{j} A\right)^{c}=E ;$ итак, $\mu\left(\left(\bigcup_{j=0}^{n-1} T^{j} A\right)^{c}\right)=0$.

\section{2. Разложение $\mathbb{R}^{n}$. Положим}

$$
E_{u}=\left\{a=\left(a_{1}, \ldots, a_{n}\right) \in \mathbb{R}^{n}: \exists a_{i_{1}}, \ldots, a_{i_{l}}, \beta_{1}, \ldots, \beta_{l}= \pm 1, \sum_{j=1}^{l} \beta_{j} a_{i_{j}}=l u\right\}
$$

и $F_{u}=E_{u}^{c}$. При $a \in F_{u}$ мы введем $u$-разложения $a$, которые разделяют $R_{t}(a)$ на правую и левую части, одна из которых может быть тривиальной. Рассуждения, подобные использованным в доказательстве леммы 5.1.1, показьвают, что имеется единственная пара $\left(i, c^{*}\right) \in\{0,1, \ldots, n\} \times \mathbb{R}$, удовлетворяюшая условию $R_{t}(a)>u t+c^{*}$ для всех $t \in[0, n] \backslash\{i\}$ и условию $R_{i}(a)=u i+c^{*}$. Иначе говоря, имеется единственная прямая с наклоном $u$, для которой $\mathrm{co}(a)$ лежит вьше этой прямой, за исключением одной точки $\left(i, R_{i}(a)\right)$, где прямая пересекает со $(a)$. Пусть $I_{u}: F_{u} \rightarrow\{0,1, \ldots, n\}$ сопоставляет каждому $a \in F_{u}$ момент $i$ только что описанного единственного пересечения. Это $u$-разложение $a \in F_{u}$ определяет разбиение $R_{t}(a)$ в точке $I_{u}(a)$ на левую и правую части, одна из которых может быть пуста или тривиальна (такое бывает при $I_{u}(a)=0$ или $\left.n\right)$. Правая часть этого разбиения удовлетворяет условию $a_{I_{u}(a)+1}+\cdots+a_{I_{u}(a)+i}>u i$ при $1 \leqslant i \leqslant n-I_{u}(a)$, в то время как левая часть этого разбиения удовлетворяет условию $-\left(a_{I_{u}(a)}+\cdots+a_{I_{u}(a)-j}\right)>-(j+1) u$ при $0 \leqslant j<I_{u}(a)$. Единственность $u$-разложения приводит к следующему разбиению $F_{u}$ : $F_{u}=\bigcup_{j=0}^{n} \mathscr{D}_{j}^{u}, \mathscr{D}_{j}^{u} \cap \mathscr{D}_{l}^{u}=\varnothing$ при $j \neq l$, где

$$
\begin{aligned}
\mathscr{D}_{j}^{u}= & \left\{a \in F_{u}: I_{u}(a)=j\right\} \\
= & \left\{a \in F_{u}: a_{j+1}+\cdots+a_{j+i}>u i, 1 \leqslant i \leqslant n-j,\right. \\
& \left.a_{j}+\cdots+a_{j-l}<(l+1) u, 1 \leqslant l<j\right\} \\
= & \left\{a \in F_{u}: a_{j+1}+\cdots+a_{j+i}>u i, 1 \leqslant i \leqslant n-j\right\} \\
& \quad \cap\left\{a \in F_{u}: a_{j}+\cdots+a_{j-l}<(l+1) u, 0 \leqslant l<j\right\} .
\end{aligned}
$$

Это разбиение $F_{u}$ сейчас поможет нам вычислить одну полезную величину. Пусть $X_{1}, \ldots, X_{n}$ - вешественные независимые одинаково распределенные случайные величины, распределения которых имеют плотности, и пусть Р обозначает совместную вероятностную меру, индуцированную $X_{1}, \ldots, X_{n}$; мы не предполагаем существования моментов. Определим $\mathrm{P}^{\prime}$ как совместное вероятностное распределение на $\mathbb{R}^{n}$, порожденное этими случайными величинами. Для $u \in \mathbb{R}$ положим $G_{j}(u)=\mathrm{P}\left(X_{1}+\right.$ $\left.\cdots+X_{i}>u i, 0<i \leqslant j\right)$ при $j=1, \ldots, n$, и пусть $G_{0}(u)=1$; положим также $K_{j}(u)=\mathrm{P}\left(X_{1}+\cdots+X_{i}<u i, 0<i \leqslant j\right)$ при $j=1, \ldots, n$, и пусть $K_{0}(u)=1$.

ТеОрема 5.2.1. $\sum_{j=0}^{n} K_{j}(u) G_{n-j}(u)=1$ для любого $u \in \mathbb{R}$. 
ДокаЗАТЕльство. Фиксируем $u \in \mathbb{R}$. Пусть $\mathscr{L}$ - лебегова мера на $\mathbb{R}^{n}$. Так как $\mathrm{P}^{\prime} \ll \mathscr{L}$ и $\mathrm{P}^{\prime}\left(E_{u}\right)=0$, то $\mathrm{P}^{\prime}\left(F_{u}\right)=1$, поскольку $E_{u}-$ конечное объединение подмногообразий коразмерности один (гиперплоскостей) в $\mathbb{R}^{n}$, и поэтому оно имеет нулевую лебегову меру. Так как $F_{u}=\bigcup_{j=0}^{n} \mathscr{D}_{j}^{u}$ и множества $\left\{\mathscr{D}_{j}^{u}\right\}_{j=0}^{n}$ попарно не пересекаются, то $\sum_{j=0}^{n} \mathrm{P}\left(\mathscr{D}_{j}^{u}\right)=1$. Следующая цепочка вычислений доказьвает теорему:

$$
\begin{aligned}
\mathrm{P}^{\prime}\left(\mathscr{D}_{j}^{u}\right)= & \mathrm{P}^{\prime}\left(a \in F_{u}: a_{j+1}+\cdots+a_{j+i}>u i, 1 \leqslant i \leqslant n-j\right) \\
& \times \mathrm{P}^{\prime}\left(a \in F_{u}: a_{j}+\cdots+a_{j-l}<u(l+1), 0 \leqslant l<j\right) \\
= & G_{n-j}(u) K_{j}(u) .
\end{aligned}
$$

Первое равенство следует из того факта, что $\mathrm{P}^{\prime}$ - произведение мер, а второе справедливо потому, что $\mathrm{P}^{\prime}$ - индуцированное произведение мер на $\mathbb{R}^{n}$, порожденное независимыми одинаково распределенными случайными величинами, и потому инвариантное относительно перестановок координат. Это завершает доказательство.

5.3. $W\left(\partial \mathscr{M}_{s}^{r}\right)=0$. В этом приложении обсуждается равенство $W\left(\partial \mathscr{M}_{s}^{r}\right)=0$. Во-первых, равномерно разобьем единичный отрезок на $2^{n}$ частей. Поставим следуюший вопрос: пусть известно, что броуновские траектории принимают значения в $\left[-2^{-n}, 2^{-n}\right]$ в момент времени 1 и лежат вьше прямой, соединяюшей начало координат с конечной точкой (в момент времени 1) в каждой точке вида $i 2^{-n}$ при $i=$ $1, \ldots, 2^{n}-1$; чему равна вероятность того, что броуновская траектория принимает значения в $\left[-2^{-n}, 2^{-n}\right]$ в некоторьй момент времени $j 2^{-n}$, где $j=1,2, \ldots, 2^{n}-1$ ? Это - простое вычисление при известных конечномерных распределениях броуновского движения и уже доказанных результатах раздела 2:

$$
\begin{gathered}
\sum_{j=1}^{2^{n}-1} \frac{\frac{1}{j\left(2^{n}-j\right)} \int_{-2^{-n}}^{2^{-n}} \frac{\exp \left(-\frac{x^{2}}{2 j 2^{-n}}\right)}{\sqrt{2 \pi j 2^{-n}}} d x \int_{-2^{-n}}^{2^{-n}} \frac{\exp \left(-\frac{y^{2}}{2\left(1-j 2^{-n}\right)}\right)}{\sqrt{2 \pi\left(1-j 2^{-n}\right)}} d y}{\frac{1}{2^{n}} \int_{-2^{-n}}^{2^{-n}} \frac{\exp \left(-\frac{z^{2}}{2}\right)}{\sqrt{2 \pi}} d z} \\
\leqslant \sum_{j=1}^{2^{n}-1} \frac{2}{2^{2 n}}\left(\frac{j}{2^{n}}\left(1-\frac{j}{2^{n}}\right)\right)^{-3 / 2} \rightarrow 0 \text { при } n \rightarrow \infty .
\end{gathered}
$$

Последнее выражение может быть получено с помошю аппроксимации римановскими суммами. Наше вычисление показьвает, что на самом деле все высказанные вьше утверждения справедливы. При изучении $\partial \mathscr{M}_{s}^{r}$ мы видим, что мы имеем дело именно с нужными типами вероятностей.

\section{4. Неравенство Марцинкевича-Зигмунда и большие отклонения.}

В этом приложении мы формулируем неравенство, принадлежащее Марцинкевичу и Зигмунду, доказательство которого использует неравенство Хинчина и приведено в [6]. Мы используем это неравенство для оценки $p$-х моментов случайной величины $\left|\sum_{i=1}^{n} v_{i}\right|$, где $v_{1}, \ldots, v_{n}$ - вещественные независимые одинаково распределенные случайные величины с $\mathrm{E} v_{i}=0$ и с конечньм $p$-м моментом; как обычно, $\mathrm{E} v_{i}$ обозначает математическое ожидание $v_{i}$. 
Теорема 5.4 .1 (Марцинкевич-Зигмунд). Пусть $\left\{X_{n}, n \geqslant 1\right\}-$ независимые случайные величины $c \mathrm{E} X_{n}=0$. Для любого $p \geqslant 1$ существуют положительнье постоянные $A_{p}, B_{p}$, зависящие только от р и удовлетворяющие условиям

$$
A_{p}\left\|\left(\sum_{i=1}^{n} X_{i}^{2}\right)^{1 / 2}\right\|_{p} \leqslant\left\|\sum_{i=1}^{n} X_{i}\right\|_{p} \leqslant B_{p}\left\|\left(\sum_{i=1}^{n} X_{i}^{2}\right)^{1 / 2}\right\|_{p}
$$

Вот следствие, которое дает нужньй нам результат.

СлЕДСТВИЕ 5.4.1. Пусть $\left\{X_{n}, n \geqslant 1\right\}$ - независимые одинаково распределенные случайные величины с $\mathrm{E} X_{i}=0 u \mathrm{E}\left|X_{1}\right|^{p}<\infty$, где $p \geqslant 2$. Пусть $S_{n}=\sum_{i=1}^{n} X_{i}$. Тогда $\mathrm{E}\left|S_{n}\right|^{p}=O\left(n^{p / 2}\right)$.

ДОКАЗАТЕЛЬСТВО СЛЕДСТВИЯ. При $p>2$ имеем

$$
\sum_{i=1}^{n} X_{i}^{2} \leqslant n^{(p-2) / p}\left(\sum_{i=1}^{n}\left|X_{i}\right|^{p}\right)^{2 / p}
$$

ввиду неравенства Гёльдера, и утверждение следствия вытекает из неравенства Марцинкевича-Зигмунда.

Используя это следствие и неравенство Чебышева, легко видеть, что справедливы все неравенства в разделах 2 и 4; все они являются неравенствами больших отклонений, что отражено в названии этого приложения.

\section{СПИСОК ЛИТЕРАТУРЫ}

[1] M. Avellaneda, W. E. Statistical properties of shocks in Burgers turbulence // Comm. Math. Phys. 1995. V. 172. № 1. P. 13-38.

[2] R. Bass. Markov processes and convex minorants // Séminaire de Probabilités XVIII. 1982/83. Berlin: Springer-Verlag, 1984. P. 29-41. (Lecture Notes in Math. V. 1059.)

[3] G. Baxter. A combinatorial lemma for complex numbers // Ann. Math. Statist. 1961. V. 32. P. 901-904.

[4] П. Биллингсли. Сходимость вероятностных мер. М.: Наука, 1977.

[5] J.C. Bonvin, Ph. Martin, J. Piasecki, X. Zotos. Statistics of mass aggregation in a self-gravitating one-dimensional gas // J. Statist. Phys. 1998. V. 91. № 1-2. P. 177-197.

[6] Y.S. Chow, H. Teicher. Probability Theory: Independence, Interchangeability, Martingales. Berlin: Springer-Verlag, 1988.

[7] W. E, Yu.G. Rykov, Ya.G. Sinai. Generalized variational principles, global weak solutions and behavior with random initial data for systems of conservation laws arising in adhesion particle dynamics // Comm. Math. Phys. 1996. V. 177. P. 349-380.

[8] C. Goldie. Records, permutations, and greatest convex minorants // Math. Proc. Cambridge Philos. Soc. 1989. V. 106. № 1. P. 169-177.

[9] P. Groeneboom. The concave majorant of Brownian motion // Ann. Probab. 1983. V. 11. № 4. P. 1016-1027.

[10] P. A. Martin, J. Piasecki. Aggregation dynamics in a self-gravitating one-dimensional gas // J. Statist. Phys. 1996. V. 84. № 3-4. P. 837-857.

[11] J. Pitman. Remarks on the convex minorant of Brownian motion // Progr. Probab. Statist. 1983. V. 5. P. 219-227.

[12] L. A. Shepp, S. P. Lloyd. Ordered cycle lengths in a random permutation // Trans. Amer. Math. Soc. 1966. V. 121. P. 340-357. 
[13] E. Sparre Andersen. On the fluctuations of sums of random variables. II // Math. Scand. 1954. V. 2. P. $195-223$.

[14] F. Spitzer. A combinatorial lemma and its application to probability theory // Trans. Amer. Math. Soc. 1956. V. 82. P. 323-339.

[15] А. М. Вершик, А. А. Шмидт. Предельные меры, возникающие в асимптотической теории симметрических групп. I // Теория вероятн. и ее примен. 1977. Т. 22. № 1. С. 72-88.

[16] Ya. B. Zeldovich. Gravitational instability: an approximate theory for large density perturbations // Astron. Astrophys. 1970. V. 5. P. 84-89.

Принстонский университет, США

Поступила в редакцию

E-mail: tmsuidan@math.princeton.edu

13.06.2001 